\title{
Superhydrophobic, photo-sterilize, and reusable mask based on graphene nanosheet-embedded carbon (GNEC) film
}

\author{
Zezhou Lin ${ }^{1}$, Zheng Wang ${ }^{2}$, Xi Zhang $^{1}(\varangle)$, and Dongfeng Diao ${ }^{1}$ \\ ${ }^{1}$ Institute of Nanosurface Science and Engineering, Guangdong Provincial Key Laboratory of Micro/Nano Optomechatronics Engineering, \\ Shenzhen University, Shenzhen 518060, China \\ ${ }^{2}$ Shenzhen Anhio Medical Technology Co., Ltd, Shenzhen 518110, China
}

(c) Tsinghua University Press and Springer-Verlag GmbH Germany, part of Springer Nature 2020

Received: 21 August 2020 / Revised: 29 September 2020 / Accepted: 4 October 2020

\section{ABSTRACT}

The 2019 coronavirus disease (COVID-19) has affected more than 200 countries. Wearing masks can effectively cut off the virus spreading route since the coronavirus is mainly spreading by respiratory droplets. However, the common surgical masks cannot be reused, resulting in the increasing economic and resource consumption around the world. Herein, we report a superhydrophobic, photo-sterilize, and reusable mask based on graphene nanosheet-embedded carbon (GNEC) film, with high-density edges of standing structured graphene nanosheets. The GNEC mask exhibits an excellent hydrophobic ability (water contact angle: $157.9^{\circ}$ ) and an outstanding filtration efficiency with $100 \%$ bacterial filtration efficiency (BFE). In addition, the GNEC mask shows the prominent photo-sterilize performance, heating up to $110^{\circ} \mathrm{C}$ quickly under the solar illumination. These high performances may facilitate the combat against the COVID-19 outbreaks, while the reusable masks help reducing the economic and resource consumption.

\section{KEYWORDS}

COVID-19, graphene nanosheet, superhydrophobic, photo-sterilize

\section{Introduction}

Coronavirus disease 2019 (COVID-19) has spread to more than 200 countries and regions $[1,2]$. By $27^{\text {th }}$ September 2020 , the number of confirmed cases has surpassed 32,730,945 worldwide with 991,224 deaths, according to data compiled by World Health Organization (WHO) [3]. Coronavirus spread through direct or indirect contact, respiratory droplets (including larger droplets that fall rapidly near the source as well as coarse aerosols with aerodynamic diameter $>5 \mu \mathrm{m}$ ), and fine-particle aerosols (diameter $\leq 5 \mu \mathrm{m}$ ) [4, 5]. Coronavirus is highly contagious and can be transmitted through respiratory droplets and contact with infected persons or fomites [6]. Droplet refers to the saliva and snot from the ill individuals talking, coughing, or sneezing. Surgical face masks could prevent transmission of human coronaviruses and influenza viruses from symptomatic individuals [7-9]. Nevertheless, the current surgical face masks still have some limitations [10-12]. Firstly, although the common mask filters the main dust particles $(>10 \mu \mathrm{m})$, the bacteria and virus filtration efficiency still need to be improved. Secondly, the lack of superhydrophobic properties caused the droplets (carrying virus) remain on the surface. Thirdly, the mask without photo- sterilize cannot be used for a long time or repeatedly, since the absorbed viruses might survive on their surfaces. Shortage of face masks is a current critical concern since the emergence of pandemic [13]. The increasing demand for masks, the large number of raw materials consumed in the preparation, and the disposal of a large number of discarded masks are all issues of great concern.
Coronavirus can hardly be transmitted by itself and requires respiratory droplets as the virus carrier [8]. Surgical masks could block the droplets containing the virus by reducing the amount and speed of droplets, and prevent the wearer from breathing in them. The peculiar properties of superhydrophobic coatings (water contact angle $>150^{\circ}$ ) [14], such as self-cleaning, anti-bacteria, and so on, are the most dramatic $[15,16]$. During recent research, microstructures have accelerated the progress of the superhydrophobic surfaces. Common polymers, such as polystyrene, polyethylene, polypropylene [17-19], heterogeneous graphene [20], and nano-hierarchical structures [21], have been prepared as superhydrophobic and anti-virus coatings. Nevertheless, reports on surgical masks with superhydrophobic ability are still rare.

Here, we reported a superhydrophobic, photo-sterilize, and reusable mask that can be worn for a long time and reused after solar illumination. The mask is based on graphene nanosheetembedded carbon (GNEC) film and developed by ultrasonicextrusion, to provide protection against the COVID-19. In hydrophobicity and photothermal properties, GNEC masks show better performance (water contact angle: $157.9^{\circ}$, temperature: $110{ }^{\circ} \mathrm{C}$ ) than laser-induced graphene (silver nanoparticles) coatings (water contact angle: $140^{\circ}$, temperature: $\sim 90^{\circ} \mathrm{C}$ ) [22].

\section{Results and discussion}

Superhydrophobic surfaces for surgical masks can be realized by using the ultrasonic extrusion method to embed GNEC 
nanostructure in smooth fibers (polypropylene, chemical structural formula: $\left.-\left[\mathrm{CH}_{2}-\mathrm{CH}(\mathrm{CH})_{3}\right]_{n}-\right)$. As shown in Fig. 1(a), the GNEC films were deposited on Si substrate by using electron cyclotron resonance (ECR) sputtering system (see Fig. S1 and Experimental Section in the Electronic Supplementary Material (ESM) for details). Low energy electrons exchange energy with valence electrons of carbon atoms through inelastic scattering, and induce the change of $\mathrm{C}-\mathrm{C}$ hybridization mode from $\mathrm{sp}^{3}$ to $\mathrm{sp}^{2}$. During one inelastic scattering process of irradiating electron, its kinetic energy is lost through several means (electron excitation, plasmon excitation, phonon excitation, etc.). When the energy loss is large enough, one part of it $\Delta E$ can trigger the hybridization change of carbon atom by breaking a $\mathrm{C}-\mathrm{C}$ bond in $\mathrm{sp}^{3}$ system. This increasing electron energy loss is essential for breaking the $\mathrm{C}-\mathrm{C}$ bond to form $\mathrm{sp}^{2}$ hybridization. With the assistant of the low-energy electrons, a large number of graphene nanosheets (GNs) embedded in amorphous carbon grow vertically to the substrate. Due to the large number of standing-structured GNs embedded in the film, the density of graphene edges is considerably high. The electron energy was modulated by deposited voltage $\left(V_{\text {dep }}\right)$ which affects the size and number of GNs. The detailed information of ECR apparatus and depositing process could be found in our former work [23-25]. The cross-sectional sample of $40 \mathrm{~V}$ GNEC film on the substrate was prepared by focused ion beam (FIB) etching (see Fig. S2 in the ESM for details). From the transmission electron microscope (TEM) image of the cross-sectional sample, GNs grew vertically on silicon substrate with the vertical thickness of $70 \mathrm{~nm}$. Based on a lot of our previous research, the high edge density of graphene nanosheet was fabricated by setting the substrate bias $40 \mathrm{~V}$ (see Fig. S3 in the ESM for details).

Figure 1(b) illustrates the fabrication process of GNEC mask by the ultrasonic extrusion method. Specifically, the thicknesses of GNEC film is $70 \mathrm{~nm}$ controlled by the deposited time, the inset shows that the graphene nanosheets grew vertically on the $\mathrm{Si}$ substrate. Electric carving device with $11,000 \mathrm{~min}^{-1}$ high-frequency varication was used for exfoliating the GNEC film from the Si substrate. Before processing, $10 \mathrm{~mL}$ of alcohol was used for wiping the surface of GNEC/Si substrate for $10 \mathrm{~s}$ to remove the dust particles. The clamp (with rubber head) was used for fixing the GNEC/Si substrate. When the carving device power is stabilized at $50 \mathrm{~W}$, the substrate vibrates evenly to peel off the GNEC film fragments. The collected GNEC fragment was uniformly and randomly distributed on the melt-blown fibers (area: $17.2 \mathrm{~cm} \times 9.2 \mathrm{~cm}$ ), which are made of thermoplastics such as polypropylene [26]. A handful of silicon doped graphene may provide a considerably carrier mobility [27]. The ultrasonic extrusion platform was used to embed GNEC nanostructure in smooth fibers. The extrusion time is $10 \mathrm{~s}$ with the ultrasonic frequency of $40 \mathrm{kHz}$ and the working power of $600 \mathrm{~W}$ (see Fig. S4 in the ESM for details). Unlike the smooth fibers of pristine surgical mask, the fibers of new mask were covered by GNEC film fragments randomly and evenly. During the ultrasonic extrusion process, the graphene nanosheets are not required to grow vertically to the fiber, since the GNEC film fragment has already contained a high density of graphene edges due to its unique nanostructure.

Figure 2(a) shows the principle of hydrophobicity caused by electron trapping at edges of GNs. The repulsive force between GNEC film and water droplet was enhanced by the surface charges. The edge quantum wells serve as electron trapping centers. In the Hubbard model, the spin-polarized Hamiltonian of graphene $p_{z}$ electron can be expressed as [28]:

$$
\begin{aligned}
& H_{\sigma}= \sum_{i, \sigma} \Delta_{i} c_{i, \sigma}^{+} c_{i, \sigma}+\sum_{<i, j>, \sigma} t_{i j}\left(f_{i j} c_{i, \sigma}^{+} c_{j, \sigma}+f_{i j}^{*} c_{j, \sigma}^{+} c_{i, \sigma}\right)+ \\
& \sum_{i} U_{i} \hat{n}_{i, \sigma} \hat{n}_{i,-\sigma} \\
& t_{i j}=<i\left|V_{i}\right| j>
\end{aligned}
$$

where $i$ represents the $i$-th atom, $j$ represents the nearest neighbor, $\sigma$ is the spin with sign, $t_{i j}$ is the overlap integral between the nearest neighbors, $f_{i j}$ is the periodic factor determined by the structure, $c_{i, \sigma}^{+}$and $c_{i, \sigma}$ are the creation and annihilation operator in Wannier representation, and $n_{i, \sigma}=c_{i, \sigma}^{+} c_{i, \sigma}$ represents the

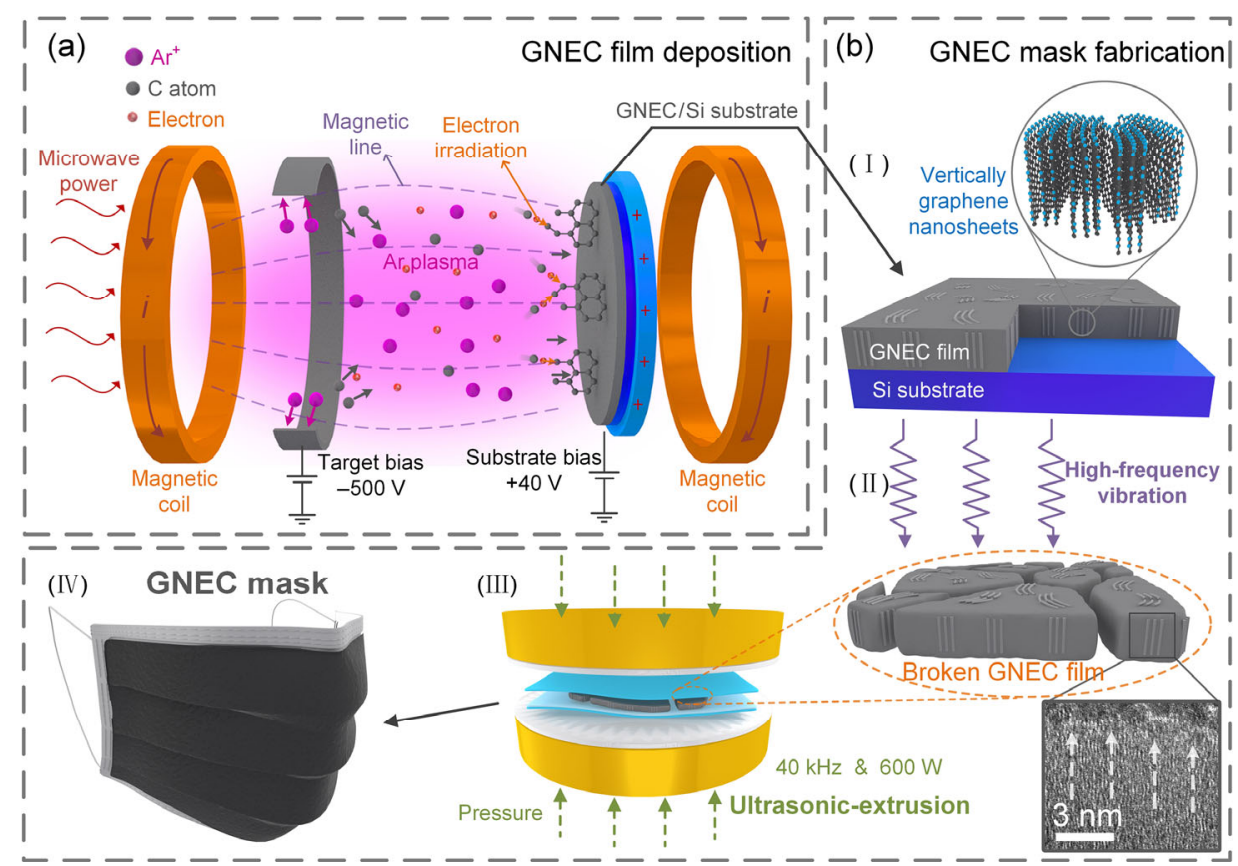

Figure 1 (a) Deposition process of GNEC film, the GNEC film was deposited on silicon substrate. (b) Fabrication process of the GNEC mask. (I) The deposited GNEC film with vertically grown graphene nanosheets with the thickness of $70 \mathrm{~nm}$. (II) GNEC film was broken by high-frequency vibration. (III) Ultrasonic-extrusion of GNEC film with $40 \mathrm{kHz}$ and $600 \mathrm{~W}$, the GNEC fragment was uniformly distributed between the melt-blown fibers. (IV) A three-dimensional diagram of the finished GNEC mask. 

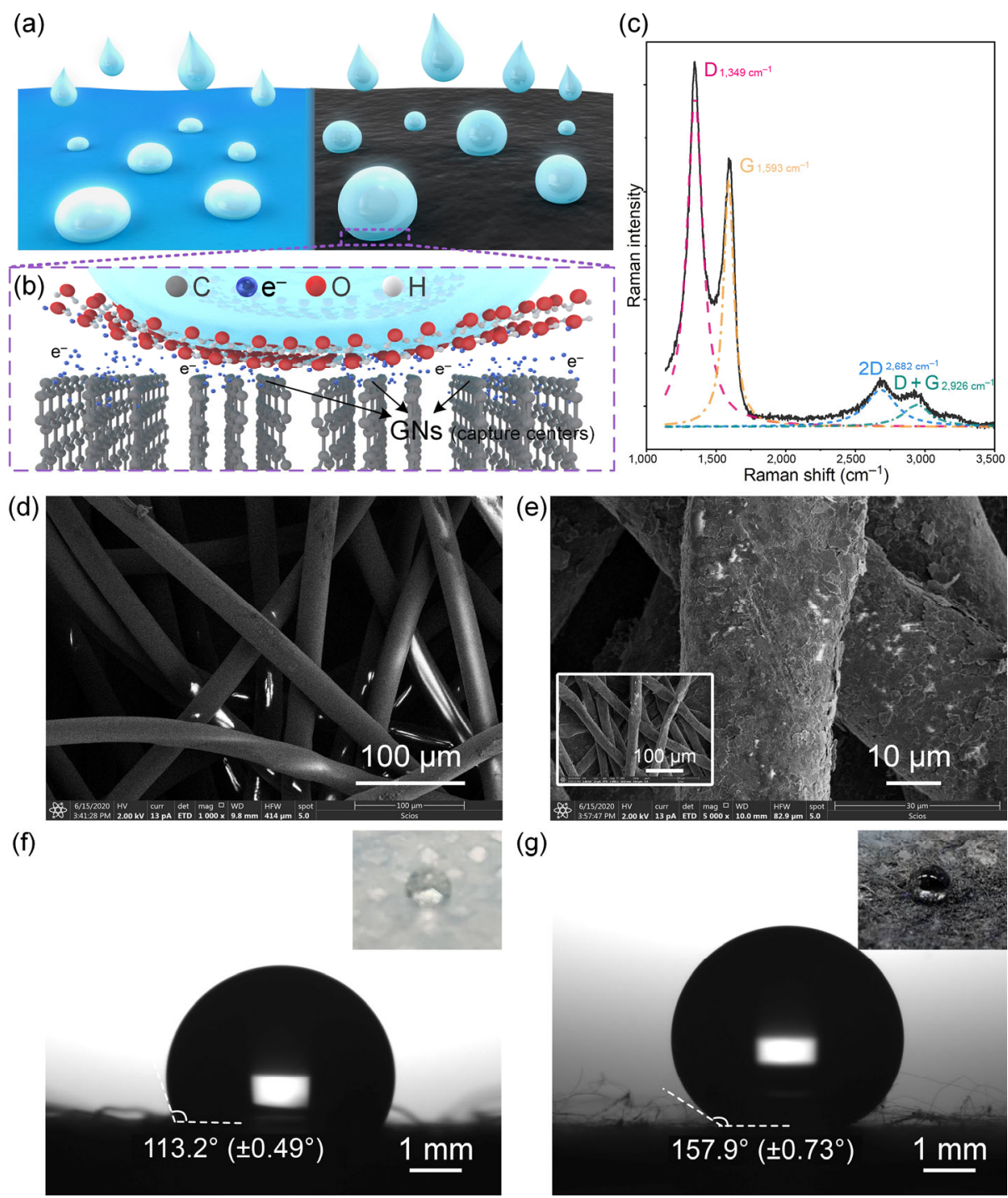

(g)

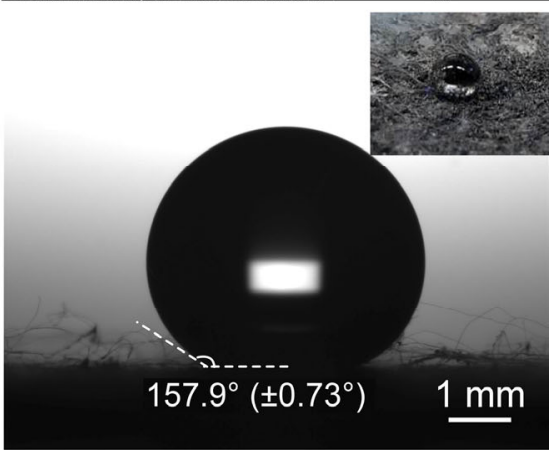

Figure 2 (a) Schematic illustration of the surface of pristine mask and GNEC mask. (b) Intrinsic mechanism of the superhydrophobic property of GNEC mask. (c) Raman spectrum of the GNEC mask. SEM of the smooth fiber of pristine mask (d) and the graphene nanosheets embed nonwoven fiber within the GNEC mask (e), the inset is a zoom-out image. Water contact angle measurement of pristine mask (f) and GNEC mask (g), with a volume of $5 \mu \mathrm{L}$ water.

particle-number operator. The diagonal energy $\Delta_{i}$ is the onsite integral. $U_{i}$ is the Hubbard repulsive energy. $V_{i}$ is the effective atomic potential.

At edges, the effective coordination number decreases, and the Hamilton matrix can be corrected as

$$
\begin{cases}C_{z}(z)=d_{i}\left(z_{i}\right) / d_{i B} & \text { (Bond contraction) } \\ C_{z}^{-m}=\frac{E_{i}\left(z_{i}\right)}{E_{i B}}=\frac{V\left(z_{i}\right)}{V_{B}} & \text { (Bond energy; potential) } \\ \frac{t\left(z_{i}\right)}{t_{B}}=\frac{<\phi_{i}(r)\left|V_{i}\left(z_{i}\right)\right| \phi_{i}(r)>}{<\phi_{i}(r)\left|V_{i B}\right| \phi_{i}(r)>} \cong & \\ \frac{V_{i}\left(z_{i}\right)}{V_{i B}} \cong C_{z}^{-m} & \text { (Hopping integral) } \\ U_{i}\left(z_{i}\right) / U_{j}\left(z_{j}\right)=\left[C_{z}\left(z_{i}\right) / C_{z}\left(z_{j}\right)\right]^{-3} & \text { (Repulsion energy) }\end{cases}
$$

where the effective coordination number $(z)$, bond length $(d)$, the indicator for bond nature of carbon $(m)$, the wave function $\left(\phi_{i}(r)\right)$, bond contraction coefficient $\left(C_{z}\right)$, single bond energy $(E)$, atomic potential $\left(V_{i}\right)$, Hamiltonian integrals $t$, and Hubbard $U$ were taken into consideration. The modification of under-coordinated is with respect to the bulk values $(B)$.
At the edge of GN, bond length $\left(d_{i}\right)$ was shortened by $C_{z}$ compared with that of bulk $\left(d_{0}\right)$ due to the automatically relaxation towards lower energy [29]. Equation (2) indicated that the effective atomic potential $\left(V_{i}\right)$ to neighbor electrons at the edges was lowered (by $C_{z}^{-m}$ ) compared with those in bulk, which tends to trap the excess electrons $[29,30]$ and unpaired spins $[29,31]$. In GNEC film, a large number of standing structured GNs provide high density of edges and trapped electrons. The surface aggregated charges of GNEC film make the mask hydrophobic.

The microstructural and physical properties of the GNEC mask are systematically characterized. In the Raman spectrum of the GNEC film, the D peak, the G peak, and the 2D peak were used to characterize the structural defects and edges, the number of layers of the GN, and the stacking pattern of the graphene layer. The ratio of the $\mathrm{D}$ peak/G peak $I_{\mathrm{D}} / I_{\mathrm{G}}$ is used to indicate the edge structure of the GNEC film, and the $2 \mathrm{D}$ peak is used to indicate the crystallinity of the GNEC film [32]. The nanostructures of graphene nanosheet have been verified by Raman spectra in Fig. 2(c). A strong 2D peak around 2,682 $\mathrm{cm}^{-1}$ appears and the $I_{\mathrm{D}} / I_{\mathrm{G}}$ is 1.38 , indicating the formation of smallsize graphene nanosheets embed in the smooth fibers.

As shown in Fig. 2(d), scanning electron microscopy (SEM) was used for observing the micro-structures of pristine mask. 
The nonwoven polymer, is composed of melt-blown fibers (diameter: $25 \mu \mathrm{m}$ ), which are distributed randomly and cross each other. Figure 2(e) displays SEM images of GNEC mask after ultrasonic-extrusion. Unlike the smooth fibers of pristine surgical mask, GNEC mask was randomly and evenly covered with graphene nanosheets nanostructure. Ultrasonic extrusion process and the optical image of the pristine mask and GNEC mask were shown in Fig. S5 in the ESM. Details of equipment and setting used for water contact angle can be found in the Experimental Section in the ESM. Coronavirus can hardly be transmitted by itself and requires respiratory droplets as the virus carrier. As shown in Fig. 2(f), a static water contact angle was used for investigating the wetting state of the pristine mask. The contact angle of $5 \mu \mathrm{L}$ water on the pristine mask surface is about $113.2^{\circ}\left( \pm 0.49^{\circ}\right)$, which is the average of the three locations of the same mask and each location was measured 30 times (measuring time: $15 \mathrm{~s}$, frequency: $2 \mathrm{~Hz}$ ). Multiple measurements show the weak hydrophobic property of pristine mask, resulting from the surface of the nonwoven polymer, is smooth and lacks nanostructures. Although weak hydrophobic properties are possessed, virus droplets still probably adhere to the surface of the pristine mask. After ultrasonic-extrusion, graphene nanosheets embedded nanostructures on the surface of the fibers improving the hydrophobic properties. Superhydrophobic properties reduce the amount of droplets adsorption facilitating medical application. The surface chemistry, material molecular weight, and structure are important in this case, especially the polarity of any defect groups. In the case of graphene-based surfaces, the defects will be key determinants of the hydrophobicity and hydrophilicity $[28,33]$. As shown in Fig. 2(g), the GNEC mask exhibits excellent superhydrophobic performance, wherein the maximum contact angles of the GNEC mask can reach to $157.9^{\circ}\left( \pm 0.73^{\circ}\right)$. The super-hydrophobicity may arise from the repulsive force between GNEC film and water droplet enhanced by the surface charges trapped by edges. Superhydrophobic surface effectively prevents the respiratory droplets (containing virus) from attaching to the mask.

Generally, self-cleaning materials are cleared by rolling water droplets to keep the surface clean $[34,35]$. The rolling angle of GNEC mask is also measured as we investigate the movement of droplets on a mask that people wear daily. Figure S6 in the ESM shows the water rolling process of the GNEC mask, the tilt angle of the test platform is $0^{\circ}-90^{\circ}$ (tilt speed: $0.5^{\circ} / \mathrm{s}$ ). At a small inclined angle $\left(7.0^{\circ}\right)$ of the platform, the droplets perceptibly roll on the mask surface. As the angle continues to

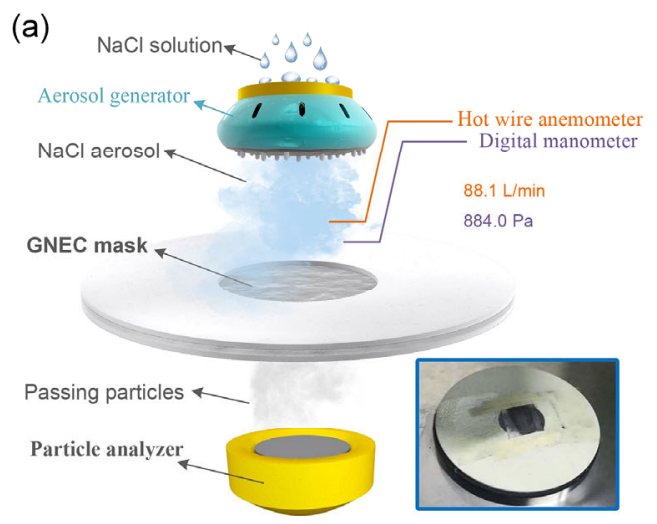

increase to $45^{\circ}$, the test drop rolled out of the measured range, indicating excellent rolling characteristics. During the daily use of masks, which should be fitted to the wearer's face instead of parallel to the ground, all places of the mask have a large inclined angle (about $90^{\circ} \pm 30^{\circ}$ ), which meets the rolling condition $\left(>7.0^{\circ}\right)$ of GNEC mask. The result indicates the potential medical application in daily life that the infected droplet (containing virus) that meets the mask will roll and slide over the surface of mask (about $60^{\circ}-120^{\circ}$ ) because the GNEC mask possesses the small rolling angle $\left(7.0^{\circ}\right)$, indicating the lower appearance of remaining droplets.

Filter efficiency measurement is a crucial step before surgical masks are commercially available. Respiratory droplets exist in different sizes, where aerosols specifically consist of droplets that are sub- $5 \mu \mathrm{m}$ in size. Droplets that are larger than $5 \mu \mathrm{m}$ generally do not spread long distances and settle within 1-2 m as a result of gravity sedimentation [36]. Masks that meet specific testing requirements can only be used for people. Bacterial Filtration Efficiency (BFE) is a common and major test item, which refers to the filtration efficiency of aerosols (diameter: $2.5 \mu \mathrm{m}$ ). In high-risk situations, mask requires more stringent measuring standards. Particulate Filtration Efficiency (PFE) is used for measuring non-oily suspended particles $(0.3 \mu \mathrm{m})$. As for the surgical mask, Technical Standard of Surgical Masks (Y0469-2011) is common measuring standard with BFE $>95 \%$ and PFE $>30 \%$.

Figure 3(a) illustrates the experimental apparatus of filter efficiency measurement. Sodium chloride $(\mathrm{NaCl})$ aerosol is often used in mask filtration efficiency measurement [37]. The $\mathrm{NaCl}$ aerosols are generated by the aerosol generator, producing particles $(0.1-10 \mu \mathrm{m})$. The air flow rate $(88.1 \mathrm{~L} / \mathrm{min})$ is measured using a hot wire anemometer and the resistance $(884.0 \mathrm{~Pa})$ is measured using a digital manometer. To prevent the experimental error caused by the movement, the prepared mask is held in place using the clamp for a better seal. The effective area of the mask sample during the tests was $\sim 100 \mathrm{~cm}^{2}$. The particle analyzer collects the passing particles and analyses the filter efficiency of different diameters particles $(10,5,2.5$, 1, 0.5, and $0.3 \mu \mathrm{m}$ ), respectively. As shown in Fig. 3(b), the pristine mask filtered $95.43 \%$ of the $2.5 \mu \mathrm{m}$ particles (BFE) and $34.68 \%$ of the $0.3 \mu \mathrm{m}$ particles (PFE), meeting the Y04692011 standards. In contrast, the GNEC mask exhibits the outstanding filter efficiency (BFE $=100 \%$ and $\mathrm{PFE}=94.01 \%)$. Compared with Figs. 2(c) and 2(e), there are large holes in the inter-crossing fibers. Pristine masks can block most particles over $2.5 \mu \mathrm{m}$. Due to the existence of holes, particles between

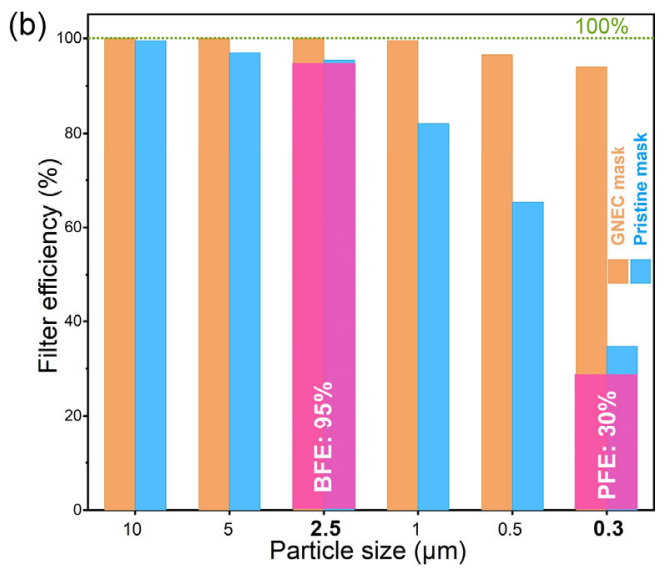

Figure 3 (a) Schematic illustration of filter efficiency measurement process. Dispersed NaCl aerosols are introduced using aerosol generator, passing through the measured mask. The passing aerosols are analyzed using particle analyzers, and the filter efficiencies are determined by collected particle concentrations. The inset shows the clamp and the testing process. (b) Mask filtration efficiency measurement. The blue represents the pristine mask and the orange represents the GNEC mask. The pink represents Technical Standard of Surgical Masks (Y0469-2011), BFE > 95\% and PFE > 30\%. 
0.3 and $2.5 \mu \mathrm{m}$ readily pass through the nonwoven fabric resulting in the low PFE. After ultrasonic extrusion, the graphene nanosheets are firmly embedded into the nanosheets filling the holes. We speculate that the enhanced performance of the hybrids is likely due to the combined effect of mechanical and trapped electron-based filtration. With the assistance of high-density graphene nanosheets, even small particles of $0.3 \mu \mathrm{m}$ are difficult to pass through the GNEC mask. The enhancement of filtration efficiency may origin from the change of microstructure of fibers caused by adding GNEC films, and can be attributed to the surface electrons trapping of GNEC film.

To further study the GNEC mask to sterilize the residual virus, the photothermal performances were investigated on the surface. Coronaviruses have a protein "ridge" (spike protein) that binds to human cells more strongly than similar viruses, enabling them to better infect and spread faster [38]. Spike proteins are sensitive to temperature, which can be inactivated by high temperatures. Recent studies have shown that under heating at $56^{\circ} \mathrm{C}$ for 30 minutes, coronavirus cannot be killed completely and researchers have found that the virus can still replicate. The thermal performance of the mask was studied by measuring the surface temperature under simulated lighting conditions with the infrared camera. As shown in Figs. 4(a) and $4(\mathrm{~b})$, the infrared camera was used to measure the temperature distribution on the surface of pristine mask and GNEC mask, under the $1,769 \mathrm{~W} / \mathrm{m}^{2}$ solar illumination (at $21 \mathrm{~V}$ input voltage) in 200 s. As shown in Fig. 4(c), the temperature of the mask stabilized around $40^{\circ} \mathrm{C}$ after $200 \mathrm{~s}$ of solar illumination (at $21 \mathrm{~V}$ input voltage), which is useless for disinfection. In contrast, the temperature of GNEC mask surface rapidly rises to $87^{\circ} \mathrm{C}\left( \pm 16.1^{\circ} \mathrm{C}\right)$ in $20 \mathrm{~s}$ and stabilizes to $105^{\circ} \mathrm{C}\left( \pm 8.4{ }^{\circ} \mathrm{C}\right)$ in $50 \mathrm{~s}$. Most viruses are inactivated over $80^{\circ} \mathrm{C}$, and the photosterilize GNEC mask can be spontaneously performed under short-time solar illumination. Additionally, under normal and mild natural light, it does not sterilize itself, so there is not worry about the wearers, which will hurt their faces. Figure 4(d) shows the optical absorption spectrum of pristine and GNEC masks. In the visible to the near infrared region ( $400-1,100 \mathrm{~nm})$, pristine mask has low optical absorption with $45 \%-60 \%$. However, the ultrasonic extruded GNEC mask showed outstanding spectral absorption performance (greater than 99.5\%) in the same range. The high absorption is beneficial for GNEC masks to show stronger photothermal performance. Compared to pristine mask, GNEC mask possesses the photo-sterilize capacity, wiping out the viruses in a short time and making the mask reusable. After 24,48 , and $72 \mathrm{~h}$ solar illumination $\left(1,262 \mathrm{~W} / \mathrm{m}^{2}\right)$, repeated experiments were conducted on the superhydrophobic and photothermal properties (at $15 \mathrm{~V}$ input voltage) of GNEC masks to determine reusability. The results of the water contact angle test (Fig. S7 in the ESM) and the surface temperature measurement (Fig. S8 in the ESM) kept their advanced performances in the superhydrophobic and photothermal features, indicating the feasibility of applications from the reused masks. Furthermore, mental clusters play an important role in antivirus effects [39]. In further studies, we will consider doping metal cluster to achieve better performance.

\section{Conclusions}

Hence, we have reported a superhydrophobic, photo-sterilize, and reusable mask based on GNEC film, thus potentially contributing to control the rapid spread of the COVID-19. GNEC films were deposited by ECR sputtering system, containing a large number of standing structured GNs. The ultrasonic extrusion method was used for fabricating the GNEC mask. GNEC mask exhibits excellent properties, including superhydrophobic (water contact angle: $157.9^{\circ}$ ), ultrahigh bacterial filtration efficiency (BFE: 100\%), and photo-sterilize (photothermal performance: $110.6{ }^{\circ} \mathrm{C}$ ). People may be inspired by this work to explore the better performance of surgical masks, together to maintain global health and development.

\section{Acknowledgements}

This work was supported by the National Natural Science Foundation of China (No. 51605306) and Shenzhen Overseas High-Level Talents Innovation and Entrepreneurship Plan (a)

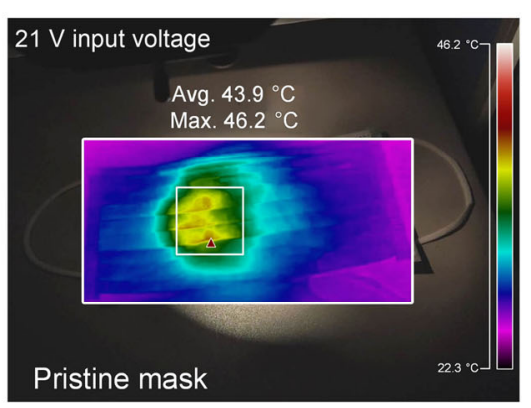

(c)

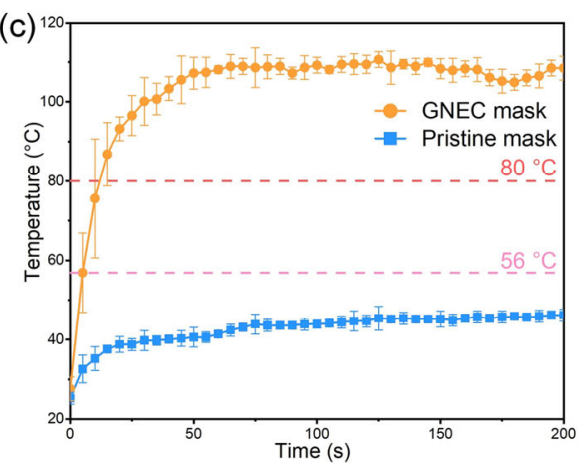

(b)

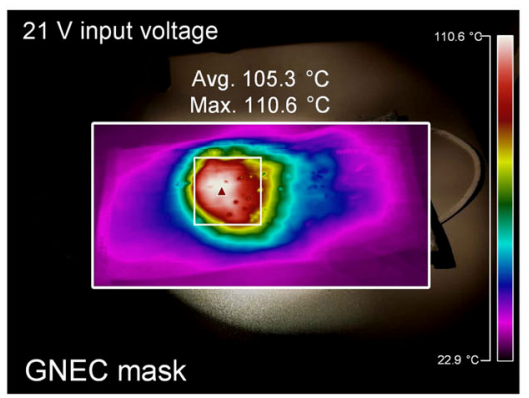

(d)

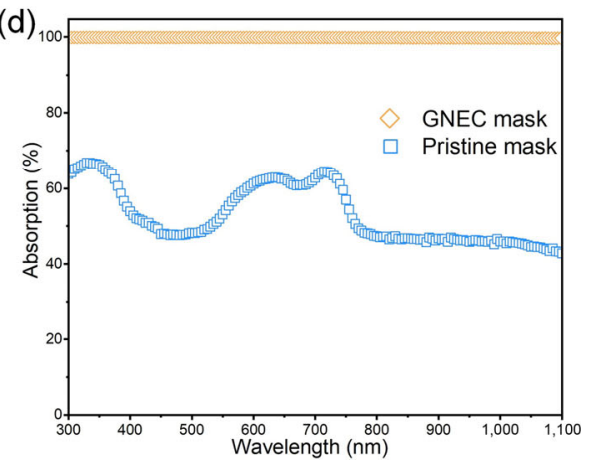

Figure 4 Photothermal performance of the masks. Surface temperature mapping of (a) a pristine surgical mask and (b) a GNEC mask after $200 \mathrm{~s}$ of solar illumination (at $21 \mathrm{~V}$ input voltage). (c) Surface temperature measurement for pristine surgical masks and GNEC masks. (d) Optical absorption of pristine and GNEC masks. 
(No. KQJSCX20180328094853770). The authors would like to thank the Electron Microscopy Center (EMC) of Shenzhen University for their technical supports in TEM and FIB.

Electronic Supplementary Material: Supplementary material (further details of electron cyclotron resonance (ECR) sputtering system, deposition of GNEC film, fabrication of GNEC mask, and characterization of the GNEC mask) is available in the online version of this article at https://doi.org/ 10.1007/s12274-020-3158-1.

\section{References}

[1] Andersen, K. G.; Rambaut, A.; Lipkin, W. I.; Holmes, E. C.; Garry, R. F. The proximal origin of SARS-CoV-2. Nat. Med. 2020, 26, $450-452$.

[2] Wang, H. W.; Wang, Z. Z.; Dong, Y. Q.; Chang, R. J.; Xu, C.; Yu, X. Y.; Zhang, S. X.; Tsamlag, L.; Shang, M. L.; Huang, J. Y. et al. Phase-adjusted estimation of the number of Coronavirus Disease 2019 cases in Wuhan, China. Cell Discov. 2020, 6, 10.

[3] WHO. Coronavirus disease (COVID-19)[Online]. https://www.who. int/docs/default-source/coronaviruse/situation-reports/20200928-we ekly-epi-update.pdf?sfvrsn=9e354665_2.

[4] Zou, L. R.; Ruan, F.; Huang, M. X.; Liang, L. J.; Huang, H. T.; Hong, Z. S.; Yu, J. X.; Kang, M.; Song, Y. C.; Xia, J. Y. et al. SARS-CoV-2 viral load in upper respiratory specimens of infected patients. New Engl. J. Med. 2020, 382, 1177-1179.

[5] Peiris, J. S. M.; Lai, S. T.; Poon, L. L. M.; Guan, Y.; Yam, L. Y. C.; Lim, W.; Nicholls, J.; Yee, W. K. S.; Yan, W. W.; Cheung, M. T. et al. Coronavirus as a possible cause of severe acute respiratory syndrome. Lancet 2003, 361, 1319-1325.

[6] Karimi, S.; Arabi, A.; Shahraki, T.; Safi, S. Detection of severe acute respiratory syndrome Coronavirus-2 in the tears of patients with Coronavirus disease 2019. Eye 2020, 34, 1220-1223.

[7] Leung, C. C.; Lam, T. H.; Cheng, K. K. Mass masking in the COVID19 epidemic: People need guidance. Lancet 2020, 395, 945.

[8] Leung, N. H. L.; Chu, D. K. W.; Shiu, E. Y. C.; Chan, K. H.; McDevitt, J. J.; Hau, B. J. P.; Yen, H. L.; Li, Y.; Ip, D. K. M.; Peiris, J. S. M. et al. Respiratory virus shedding in exhaled breath and efficacy of face masks. Nat. Med. 2020, 26, 676-680.

[9] El-Atab, N.; Qaiser, N.; Badghaish, H.; Shaikh, S. F.; Hussain, M. $\mathrm{M}$. Flexible nanoporous template for the design and development of reusable Anti-COVID-19 hydrophobic face masks. ACS Nano $\mathbf{2 0 2 0}$, 14, 7659-7665.

[10] Majchrzycka, K.; Okrasa, M.; Szulc, J.; Jachowicz, A.; Gutarowska, B. Survival of microorganisms on nonwovens used for the construction of filtering facepiece respirators. Int. J. Environ. Res. Public Health 2019, 16, 1154.

[11] Konda, A.; Prakash, A.; Moss, G. A.; Schmoldt, M.; Grant, G. D.; Guha, S. Aerosol filtration efficiency of common fabrics used in respiratory cloth masks. ACS Nano 2020, 14, 6339-6347.

[12] Zhong, H.; Zhu, Z. R.; Lin, J.; Cheung, C. F.; Lu, V. L.; Yan, F.; Chan, C. Y.; Li, G. Reusable and recyclable graphene masks with outstanding superhydrophobic and photothermal performances. ACS Nano 2020, 14, 6213-6221.

[13] Ullah, S.; Ullah, A.; Lee, J.; Jeong, Y.; Hashmi, M.; Zhu, C. H.; Joo, K. I.; Cha, H. J.; Kim, I. S. Reusability comparison of melt-blown vs nanofiber face mask filters for use in the coronavirus pandemic. ACS Appl. Nano Mat. 2020, 3, 7231-7241.

[14] Marmur, A. Hydro- hygro- oleo- omni-phobic? Terminology of wettability classification. Soft Matter 2012, 8, 6867-6870.

[15] Zhu, H.; Guo, Z. G.; Liu, W. N. Adhesion behaviors on superhydrophobic surfaces. Chem. Commun. 2014, 50, 3900-3913.

[16] Song, L. J.; Sun, L. W.; Zhao, J.; Wang, X. H.; Yin, J. H.; Luan, S. F.; Ming, W. H. Synergistic superhydrophobic and photodynamic cotton textiles with remarkable antibacterial activities. ACS Appl. Bio Mater. 2019, 2, 2756-2765.

[17] Hong, D. J.; Ryu, I.; Kwon, H.; Lee, J. J.; Yim, S. Preparation of superhydrophobic, long-neck vase-like polymer surfaces. Phys. Chem. Chem. Phys. 2013, 15, 11862-11867.

[18] Huovinen, E.; Takkunen, L.; Korpela, T.; Suvanto, M.; Pakkanen, T.
T.; Pakkanen, T. A. Mechanically robust superhydrophobic polymer surfaces based on protective micropillars. Langmuir 2014, 30, 14351443.

[19] Liu, M. L.; Luo, Y. F.; Jia, D. M. Polydimethylsiloxane-based superhydrophobic membranes: Fabrication, durability, repairability, and applications. Polym. Chem. 2020, 11, 2370-2380.

[20] Ding, G. M.; Jiao, W. C.; Wang, R. G.; Yan, M. L.; Chu, Z. M.; He, X. D. Superhydrophobic heterogeneous graphene networks with controllable adhesion behavior for detecting multiple underwater motions. $J$. Mater. Chem. A 2019, 7, 17766-17774.

[21] Si, Y. F.; Guo, Z. G. Superhydrophobic nanocoatings: From materials to fabrications and to applications. Nanoscale 2015, 7, 5922-5946.

[22] Zhong, H.; Zhu, Z. R.; You, P.; Lin, J.; Cheung, C. F.; Lu, V. L.; Yan, F.; Chan, C. Y.; Li, G. J. Plasmonic and Superhydrophobic SelfDecontaminating N95 Respirators. ACS Nano 2020, 14, 8846-8854.

[23] Zhang, X.; Lin, Z. Z.; Peng, D.; Ye, L.; Zang, J. F.; Diao, D. Edgestate-enhanced ultrahigh photoresponsivity of graphene nanosheetembedded carbon film/silicon heterojunction. Adv. Mater. Interfaces 2019, 6, 1802062 .

[24] Wang, C.; Zhang, X.; Diao, D. F. Nanosized graphene crystallite induced strong magnetism in pure carbon films. Nanoscale 2015, 7, 4475-4481.

[25] Zhang, X.; Peng, D.; Lin, Z. Z.; Chen, W. C.; Diao, D. F. Edge effect on the photodetection ability of the graphene nanocrystallites embedded carbon film coated on p-silicon. Phys. Status Solidi RRL 2019, 13, 1800511.

[26] Lee, Y.; Wadsworth, L. C. Structure and filtration properties of melt blown polypropylene webs. Polymer Eng. Sci. 1990, 30, 1413-1419.

[27] Wang, Z. G.; Li, P. J.; Chen, Y. F.; Liu, J. B.; Zhang, W. L.; Guo, Z.; Dong, M. D.; Li, Y. R. Synthesis, characterization and electrical properties of silicon-doped graphene films. J. Mater. Chem. C 2015, 3, 6301-6306.

[28] Zhang, X.; Nie, Y. G.; Zheng, W. T.; Kuo, J. L.; Sun, C. Q. Discriminative generation and hydrogen modulation of the DiracFermi polarons at graphene edges and atomic vacancies. Carbon 2011, 49, 3615-3621.

[29] Zhang, X.; Wang, C.; Sun, C. Q.; Diao, D. F. Magnetism induced by excess electrons trapped at diamagnetic edge-quantum well in multi-layer graphene. Appl. Phys. Lett. 2014, 105, 042402.

[30] Enoki, T.; Kobayashi, Y.; Fukui, K. I. Electronic structures of graphene edges and nanographene. Int. Rev. Phys. Chem. 2007, 26, 609-645.

[31] Ding, D.; Dai, X. Z.; Wang, C.; Diao, D. F. Temperature dependent crossover between positive and negative magnetoresistance in graphene nanocrystallines embedded carbon film. Carbon 2020, 163, 19-25.

[32] Pimenta, M. A.; Dresselhaus, G.; Dresselhaus, M. S.; Cançado, L. G.; Jorio, A.; Saito, R. Studying disorder in graphite-based systems by Raman spectroscopy. Phys. Chem. Chem. Phys. 2007, 9, 1276-1291.

[33] Liu, X. J.; Zhang, X.; Bo, M. L.; Li, L.; Tian, H. W.; Nie, Y. G.; Sun, Y.; Xu, S. Q.; Wang, Y.; Zheng, W. T. et al. Coordination-resolved electron spectrometrics. Chem. Rev. 2015, 115, 6746-6810.

[34] Zhu, H.; Wu, L. Z.; Meng, X.; Wang, Y. G.; Huang, Y.; Lin, M. H.; Xia, F. An anti-UV superhydrophobic material with photocatalysis, self-cleaning, self-healing and oil/water separation functions. Nanoscale 2020, 12, 11455-11459.

[35] Verho, T.; Bower, C.; Andrew, P.; Franssila, S.; Ikkala, O.; Ras, R. H. A. Mechanically durable superhydrophobic surfaces. Adv. Mater. 2011, 23, 673-678.

[36] Morawska, L. Droplet fate in indoor environments, or can we prevent the spread of infection? Indoor Air 2006, 16, 335-347.

[37] Cho, H. W.; Yoon, C. S.; Lee, J. H.; Lee, S. J.; Viner, A.; Johnson, E. W. Comparison of pressure drop and filtration efficiency of particulate respirators using welding fumes and sodium chloride. Ann. Occup. Hyg. 2011, 55, 666-680.

[38] Li, F. Structure, function, and evolution of coronavirus spike proteins. Ann. Rev. Virol. 2016, 3, 237-261.

[39] Lazar, P.; Zhang, S.; Šafářová, K.; Li, Q.; Froning, J. P.; Granatier, J.; Hobza, P.; Zbořil, R.; Besenbacher, F.; Dong, M. D. et al. Quantification of the interaction forces between metals and graphene by quantum chemical calculations and dynamic force measurements under ambient conditions. ACS Nano 2013, 7, 1646-1651. 